\title{
LA PRODUCCIÓN DE TESIS DE ANTROPOLOGÍA EN LA UNIVERSIDAD ACADEMIA DE HUMANISMO CRISTIANO. APROXIMACIÓN A ALGUNAS DE SUS CARACTERISTICAS
}

\author{
The Production of Anthropology Thesis at the University Academia de Humanismo \\ Cristiano. Approach to Some of its Characteristics
}

\section{CLAUDIO ESPINOZA* ${ }^{*}$ JOSÉ VARAS**, MICHELLE ALLENDES***,

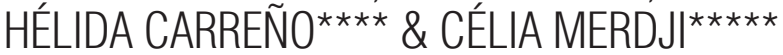

Fecha de recepción: 3 de enero de 2019 - Fecha de aprobación: 10 de mayo de 2019

\section{Resumen}

Intentando contribuir a la reflexión en torno a la producción de conocimiento en las antropologías del sur, este artículo representa un avance de resultados y tiene por objeto realizar una acercamiento descriptivo al quehacer de las ciencias antropológicas en Chile a partir de datos preliminares de un trabajo de sistematización y análisis de las tesis de grado de la Escuela de Antropología de la Universidad Academia de Humanismo Cristiano, realizadas en un período de veinte años (1998 y 2018). A partir de sus ejes temáticos, así como las zonas geográficas involucradas, se establece cierta relación entre las bases que configuraron el proyecto de formación antropológica de la escuela analizada y la producción de conocimientos reflejadas en las tesis de grado.

Palabras clave: tesis de antropología; producción de conocimientos; antropologías del sur.

\begin{abstract}
Trying to contribute to the reflection about the production of knowledge in the anthropologies of the south, this article represents a progress of results and aims to make a descriptive approach to the work of the anthropological sciences in Chile from preliminary data of a work of systematization and analysis of the theses of the department of anthropology of the University Academia de Humanismo Cristiano, carried out in a period of twenty years (1998- 2018). From its thematic axes, as well as the geographical areas involved, a certain relationship is established between the bases that configured the anthropological training project of the department analyzed and the production of knowledge reflected in the degree theses.
\end{abstract}

Keywords: anthropology thesis; knowledge production; southern anthropologies.

\footnotetext{
* Dr. en Antropología. Escuela de Antropología, Universidad Academia de Humanismo Cristiano e Investigador Asociado del Centro de Estudios Interculturales e Indígenas (CIIR), Santiago, Chile. Correo-e: cespinoza@academia.cl

** Mg. en Antropología. Encargado Unidad de Estadística y Archivo, Servicio Médico Legal, Santiago, Chile. Correo-e: jvaras@sml.gob.cl

*** Estudiante de antropología, Universidad Academia de Humanismo Cristiano, Santiago, Chile. Correo-e: michelle.allendess@gmail.com

**** Estudiante de antropología, Universidad Academia de Humanismo Cristiano, Santiago, Chile. Correo-e: hhelicoide@gmail.com

**** Estudiante de antropología, Universidad Academia de Humanismo Cristiano, Santiago, Chile.

Correo-e: celia.mdj@hotmail.fr
} 


\section{Introducción}

El presente trabajo forma parte de una investigación más amplia que busca caracterizar la producción de conocimientos antropológicos en Chile a través del estudio de las tesis de grado producidas en la Escuela de Antropología de la Universidad Academia de Humanismo Cristiano a lo largo de toda su historia. Para este primer avance de resultados se han considerado un total de 241 tesis producidas entre 1998 y 2018.

El articulo se divide en 6 partes. A continuación, se muestra la génesis del proyecto, que nace bajo la motivación de un proyecto impulsado por el profesor Roberto Melville. Luego se establece la relación entre este tipo de iniciativas y el proyecto de visibilización de las antropologías del sur, para, posteriormente hacer referencia al desarrollo de la formación antropológica institucionalizada en Chile, con una breve referencia a la historia de la escuela de antropología aquí analizada. En seguida se aborda la aproximación metodológica del estudio y la descripción de algunas de las características de las tesis analizadas. Por último, se presentan algunas consideraciones finales.

\section{El catálogo latinoamericano de tesis de antropología}

En marzo de 2017 el doctor Roberto Melville, profesor-investigador del Centro de Investigaciones y Estudios Superiores en Antropología Social (CIESAS) de México, concluyó un proyecto iniciado con las instituciones mexicanas de formación antropológica (redMIFA) consistente en la confección de un catálogo bibliográfico con todas las tesis de antropología producidas en tales centros de estudios.
El resultado fue la identificación de 7.366 tesis elaboradas entre los años 1945 y 2016.

En junio de ese mismo año se celebró en Bogotá, Colombia, el V Congreso de la Asociación Latinoamericana de Antropología (ALA), ocasión en que el profesor Melville propuso la conformación de un grupo de trabajo orientado a elaborar el catálogo latinoamericano de tesis de antropología social, buscando con ello extender a los diversos países de la región el estudio desarrollado en México.

Y así ocurrió. En octubre de 2017 recibimos un mensaje de Roberto Melville invitándonos a participar en la conformación de este catálogo e instando a la conformación de un grupo que pudiera coordinar el trabajo con las tesis de antropología producidas en las universidades chilenas. En el Congreso de Bogotá, Roberto Melville ya había reclutado para el proyecto a Héctor Mora, colega de la Universidad Católica de Temuco, quien ya llevaba tiempo trabajando con las tesis producidas en su casa de estudio (Mora, 2014), y comenzó prontamente a trabajar con las realizadas en la Universidad Austral de Chile. Una vez en relación con él, nos dimos a la tarea de contactar a colegas de otras universidades. Nos comunicamos primero con Leonardo Piña, de la Universidad Alberto Hurtado, quien, junto con comprometer el acceso a las tesis producidas en su universidad, tenía acceso a las tesis producidas en el extinto departamento de antropología de la Universidad Bolivariana. Luego fuimos ampliando el radio y nos acercamos a Andrea Chamorro, de la Universidad de Tarapacá, en el extremo norte de Chile, y a colegas de todas las universidades chilenas que brindan algún tipo de formación antropológica, ya sea en pre como posgrado. Se sumaron profesores de la Univer- 
sidad Católica del Norte y la Pontificia Universidad Católica de Chile, y esperamos que con el tiempo se puedan integrar las demás.

A través de sucesivos correos electrónicos, el profesor Melville fue compartiendo sus ideas y proyecciones con quienes nos fuimos incorporando al proyecto. Algunas de estas ideas se encuentran expuestas en la página de inicio del Catálogo Latinoamericano de Tesis en Antropología Social en línea ${ }^{1}$. Entre los diversos objetivos que tenía la elaboración de este catálogo estaba, en primer lugar, la idea de aportar a la visibilización de las contribuciones científicas realizadas por miles de estudiantes latinoamericanos, es decir, sus tesis, que son consideradas, nos recuerda Melville, como parte de lo que se conoce como literatura gris, una producción menos citada y apreciada que la producción de libros y artículos científicos. De allí la necesidad de destacar su existencia.

En segundo lugar, y dado el notable incremento de instituciones dedicadas a la formación antropológica en los últimos años, así como de la gran cantidad de egresados de dichos centros y universidades, el proyecto se proponía disponer de un instrumento actualizado que permitiera el acceso a una rica fuente de conocimientos:

\footnotetext{
Mediante la sistematización de las fuentes del conocimiento antropológico latinoamericano, ofrecemos a los estudiantes un instrumento para elaborar sus proyectos de investigación relacionándolos con trabajos que pudieran ser de su interés y han sido realizado por sus pares. Saber lo que se ha dicho y estudiado, contribuye a que nuestros conocimientos antropológicos sean cada vez más robustos y convincentes. Asimismo, el conjunto de tesis de una institución o de un país, es un reflejo de quiénes somos, qué hacemos, cuáles son nuestras inquietudes académicas, cómo cambian nuestros enfoques y qué nos hace antropólogos, a partir de nuestras propias subjetividades ${ }^{2}$.
}

Es importante también destacar la perspectiva antropológica que ha dado Melville a este proyecto, asociándolo al principio del don de Marcel Mauss: dar, recibir y devolver. Esta transmisión, cual circuito Kula, permitirá establecer redes de colaboración e intercambio entre las distintas instituciones antropológicas latinoamericanas ${ }^{3}$.

El estímulo que significó la invitación de Roberto Melville fue significativo. A partir de allí formamos un pequeño equipo de investigación, integrado por las y los autores de este artículo, e iniciamos un lento proceso de revisión de las tesis producidas en la Escuela de Antropología de la Universidad Academia de Humanismo Cristiano. Al comenzar a revisar las tesis, buscando los datos que permitirían llenar los casilleros comprendidos en el catálogo propuesto por Melville, descubrimos un mundo más amplio, que generaba nuevas preguntas y ameritaba, por tanto, una revisión más extensa, aunque, por lo mismo, más lenta y meticulosa. Pero, sin duda, muy interesante.

Así, ampliamos los aspectos a observar y fuimos revisando tesis por tesis, desde la primera, defendida en 1998, hasta la última catalogada, presentada en diciembre de 2018. Como se señaló, este proyecto está en marcha y seguimos revisando los datos y tratando de interpretarlos a la luz de diversos enfoques y escenarios. Por ello, este artículo es una primera aproximación, un avance de resultados que, junto con buscar ponerlos a discusión, pretende estimular el ejercicio aquí realizado. 


\section{Las antropologías del Sur}

Evidentemente, esta investigación, así como el trabajo liderado por el profesor Melville, se inserta en un movimiento más amplio vinculado con el trabajo realizado en torno a las antropologías del mundo (Lins Ribeiro \& Escobar, 2008), periféricas (Cardoso de Oliveira, 1997), segundas (Krotz, 2015), cociudadanas (Jimeno \& Arias, 2011) o antropologías del sur (Krotz, 1993, 2006). Todas estas aproximaciones constituyen una suma de esfuerzos y experiencias ancladas en lo que puede considerarse como una antropología de la antropología (Peirano, 1991; Díaz-Crovetto, 2011).

Este movimiento, al partir evidenciando las estructuras y relaciones de poder en las que se hallan inmersas las producciones de conocimientos y los momentos de transformaciones asociados (Lins Ribeiro \& Escobar, 2008) releva la necesidad de poner de relieve las antropologías periféricas del sistema mundo antropológico.

El silenciamiento de las antropologías del sur (Krotz, 1993) exigió el desarrollo de diversos esfuerzos para impulsar un cambio que permita visibilizar estas antropologías, entre ellas la antropología latinoamericana. Una línea de acción, propuesta por Krotz (2006), es la autorreflexión en y sobre las antropologías del sur, una antropología de las antropologías que analice las dinámicas de producción y reproducción del conocimiento antropológico.

En la búsqueda de un inventario sistemático, Krotz propone, entre otras iniciativas, buscar en sus trayectorias históricas los momentos iniciales y reconocer desde allí la dialéctica entre las hipotecas exógenas y las innovaciones endógenas, para poder distinguir los impulsos indepen- dientes generados por los "contextos" locales de los efectos directos e indirectos de la difusión del pensamiento y de las prácticas antropológicas provenientes del exterior (Krotz, 2006).

En este sentido, nuestro trabajo pretende contribuir a la reflexión en torno a la producción de conocimiento en las antropologías del sur, en particular a través del análisis de esta literatura gris: las tesis. Creemos que el estudio de esta producción antropológica puede aportar al trabajo, ya iniciado, de contrastar las diferentes antropologías latinoamericanas. Esta comparación, como señala Krotz (2006), no debe implicar el fomento de confrontaciones entre las diversas partes de la comunidad antropológica, nacional o internacional, sino más bien implica reconocer los perfiles propios en el seno de las antropologías del sur. Este reconocimiento, por otro lado, no debe significar un ensimismamiento en cada experiencia formativa, sino que debe entenderse como parte de un movimiento mayor, por ejemplo, y como es nuestro caso, articulado al trabajo de la Asociación Latinoamericana de Antropología, un espacio consagrado a la reflexión teórica del quehacer antropológico, a la convergencia de distintos saberes y al vínculo entre las diferentes comunidades antropológicas de América Latina y el Caribe (Gatti \& De Souza, 2018).

\section{La Academia de Humanismo Cristiano y la formación antropológica en Chile}

En comparación con otras tradiciones latinoamericanas (Correa, 1993; Guber, 2006; Restrepo, 2014; Vásquez, 2014), el desarrollo de la antropología en Chile tuvo una institucionalización académica tardía (Bengoa, 2014; Castro, 2014; Mora, 2016). 
Tomando en cuenta el amplio campo de las ciencias sociales, Garretón (2007) ha mostrado una periodización que nos es útil para graficar la trayectoria institucional de la formación antropológica en Chile y el desarrollo de la Escuela de Antropología aquí revisada.

El autor señala que una primera fase de creación, institucionalización y profesionalización va de mediados de la década de 1950 hasta el golpe de Estado de 1973. Esta etapa estuvo caracterizada por una gran densidad universitaria y disciplinaria, y por la forma en que el contexto sociopolítico actuó a modo de ideología intelectual dominante. A diferencia de otras disciplinas de las ciencias sociales, la institucionalización de la antropología durante este lapso fue más débil (Garretón, 2007).

Una segunda fase coincide con el período de la dictadura militar, entre 1973 y 1989, y se caracterizó por el abandono de las ciencias sociales de las universidades chilenas, hecho que se suplió a través de la creación de una serie de centros académicos independientes que albergaron su desarrollo disciplinar. Por último, una tercera fase se inició con la transición a la democracia, a partir de 1990, y se extiende, según el estudio de Garretón, hasta 20044. En esta etapa las ciencias sociales volvieron a situarse preferentemente en las universidades y se produjo una expansión de las carreras e instituciones vinculadas con ellas (Garretón, 2007).

Tal cual se observa en la Tabla № 1, la apertura de escuelas y departamentos de antropología durante la primera fase establecida por Garretón (2007) corresponde a tres universidades, dos de las cuales -Universidad de Concepción y Universidad Católica de Temuco- debieron interrumpir prontamente sus trayectorias debido al cierre obligado impuesto por la dictadura. El Departamento de Antropología de la Universidad de Chile, en cambio, tuvo continuidad durante la dictadura militar y se mantiene hasta la actualidad.

Tabla № 1: Apertura de escuelas y departamentos de antropología en universidades chilenas

\begin{tabular}{|l|l|l|l|l|} 
UNIVERSIDAD & APERTURA & CIERRE & RE APERTURA & PRIMERA FASE5 \\
\hline $\begin{array}{l}\text { Universidad de } \\
\text { Concepción }\end{array}$ & 1965 & 1973 & 2005 & \\
\cline { 1 - 3 } Universidad de Chile & 1969 & 1978 & 1992 & \multirow{2}{*}{$\begin{array}{l}\text { INSTITUCIONALIZACIÓN } \\
\text { Y PROFESIONALIZACIÓN }\end{array}$} \\
\cline { 1 - 2 } $\begin{array}{l}\text { Universidad Católica } \\
\text { de Temuco }\end{array}$ & 1973 & 1990 & \\
\hline
\end{tabular}

Fuente: Elaboración propia. 
La Tabla № 2 muestra más extensamente el tres fases de desarrollo de las ciencias sociales despliegue antropológico universitario en Chile señaladas más arriba ${ }^{6}$.

a lo largo de toda su historia, pues abarca las

Tabla № 2: Apertura y continuidad de escuelas y departamentos de antropología en universidades chilenas

\begin{tabular}{|c|c|c|c|c|}
\hline UNIVERSIDAD & APERTURA & CIERRE & PERIODO & FASES $^{7}$ \\
\hline $\begin{array}{l}\text { Universidad } \\
\text { de Chile }\end{array}$ & 1969 & & PREDICTADURA & $\begin{array}{l}\text { PRIMERA FASE: } \\
\text { INSTITUCIONALIZACIÓN } \\
\text { Y PROFESIONALIZACIÓN }\end{array}$ \\
\hline $\begin{array}{l}\text { Universidad } \\
\text { Austral de Chile }\end{array}$ & 1985 & & DICTADURA & $\begin{array}{l}\text { SEGUNDA FASE: } \\
\text { PÉRDIDA Y SALIDA } \\
\text { DE LAS UNIVERSIDADES }\end{array}$ \\
\hline $\begin{array}{l}\text { U. Academia } \\
\text { de Humanismo } \\
\text { Cristiano }\end{array}$ & 1992 & & \multirow{3}{*}{$\begin{array}{l}\text { POSDICTADURA } \\
\text { SIGLO XX }\end{array}$} & \multirow{10}{*}{$\begin{array}{l}\text { TERCERA FASE: } \\
\text { REGRESO A LAS } \\
\text { UNIVERSIDADES, NUEVAS } \\
\text { CARRERAS Y EXPANSIÓN }\end{array}$} \\
\hline $\begin{array}{l}\text { Universidad } \\
\text { Bolivariana }\end{array}$ & 1992 & 2010 & & \\
\hline $\begin{array}{l}\text { Universidad } \\
\text { Católica de } \\
\text { Temuco }\end{array}$ & $\begin{array}{l}1992 \\
\text { (reapertura) }\end{array}$ & & & \\
\hline $\begin{array}{l}\text { Universidad de } \\
\text { Tarapacá }\end{array}$ & 2005 & & \multirow{7}{*}{ SIGLO XXI } & \\
\hline $\begin{array}{l}\text { Universidad de } \\
\text { Concepción }\end{array}$ & $\begin{array}{l}2005 \\
\text { (reapertura) }\end{array}$ & & & \\
\hline $\begin{array}{l}\text { Universidad } \\
\text { Católica del Norte } \\
\text { (posgrado) }\end{array}$ & 2005 & & & \\
\hline Universidad ARCIS & 2007 & 2017 & & \\
\hline $\begin{array}{l}\text { Universidad } \\
\text { Alberto Hurtado }\end{array}$ & 2011 & & & \\
\hline $\begin{array}{l}\text { Pontificia } \\
\text { Universidad } \\
\text { Católica }\end{array}$ & 2013 & & & \\
\hline $\begin{array}{l}\text { Universidad } \\
\text { de Los Lagos }\end{array}$ & 2019 & & & \\
\hline
\end{tabular}

Fuente: Elaboración propia. 
Como señalamos, solo la Universidad de Chile pudo mantener su continuidad desde su creación en 1969, en la etapa de predictadura, hasta la actualidad. Luego, durante la segunda fase, solo se creó un departamento de antropología, el de la Universidad Austral de Chile en 1985.

Esto significa que la mayor parte de las escuelas y departamentos de antropología actualmente existentes en Chile fueron abiertos (o reabiertos) durante la tercera fase, esto es, luego de terminada la dictadura militar. Entre las primeras universidades en abrir o reabrir programas de antropología en este período están la Universidad Academia de Humanismo Cristiano, la Universidad Católica de Temuco y la Universidad Bolivariana, que abrieron esta carrera en 1992. Las dos primeras continúan hasta la actualidad, mientras que la Universidad Bolivariana cerró su departamento de antropología en 2010.

Todos los demás programas de antropología abrieron durante la década de 2000 y 2010: la Universidad de Tarapacá y la Universidad de Concepción en 2005; la Universidad Católica del Norte (posgrado) en 2005; la Universidad ARCIS en 2007 (cerrado en 2017); la Universidad Alberto Hurtado en 2011; la Pontificia Universidad Católica de Chile en 2013 y, por último, el más reciente, abrió en 2019 en la Universidad de los Lagos.

A la luz del derrotero seguido por la antropología chilena, la historia de la Escuela de Antropología de la Universidad Academia de Humanismo Cristiano refleja con bastante claridad la relación entre el contexto sociopolítico y su trayectoria.
Los antecedentes de esta universidad se remontan al 15 de noviembre de 1975 cuando, por decreto canónico, fue creada la Academia de Humanismo Cristiano por el cardenal Raúl Silva Henríquez. En esta instancia convergieron una serie de intelectuales y profesores universitarios que, después del golpe de Estado de 1973, habían sido expulsados de las universidades públicas. Con el tiempo, y a medida que se abrían las posibilidades para el retorno de exiliados, esta institución, aún no convertida en universidad, fue reclutando a más y más profesionales de uno de los campos más golpeado por la dictadura: las ciencias sociales.

Un caso representativo lo constituye el profesor José Bengoa, quien después del golpe de Estado fue exonerado de la Universidad de Chile y exiliado del país y, a su regreso, se incorporó a la Academia de Humanismo Cristiano (Espinoza, Campos \& Contreras, 2019). Más tarde, en los primeros años de la transición, y cuando la Academia ya se había constituido como universidad, se abrieron diversas carreras en el área de las ciencias sociales, entre ellas la de antropología, de la que Bengoa fue fundador y primer director.

De esta manera, la Academia de Humanismo Cristiano fungió como un centro de acogida para los expulsados de las universidades durante la dictadura y permitió el desarrollo disciplinar en una época en extremo complicada para el desarrollo del pensamiento crítico. En paralelo, convergieron alrededor de esta instancia una serie de otros centros de estudios independientes y organizaciones no gubernamentales (ONG) que, formal o informalmente, fueron dialogando entre sí. Estos centros se asociaron a temáticas y disciplinas especializadas y resultaron fundamentales para la transformación de la Academia en universidad. 
En 1987, casi al final de la dictadura, cuando la Academia pasó a conformarse como universidad, algunos de estos centros estuvieron en la base de la formación de las carreras. El caso más emblemático, y que marca de algún modo el sello de la universidad, fue la relación entre la Fundación de Ayuda de las Iglesias Cristianas (FASIC), institución que jugó un papel clave en la promoción y la defensa de los derechos humanos durante la dictadura, y la Escuela de Derecho de la Universidad.

Para el caso de la Escuela de Antropología, podríamos sostener que hubo tres centros que estuvieron en la base de su constitución y que, como veremos más adelante, marcan, hasta el día de hoy, cierta orientación en la producción científica reflejada en las tesis. Estos centros son: el WUS (Servicio Universitario Mundial, en español), que trabajaba fuertemente por la promoción educacional entre los líderes sociales, muchos de ellos líderes indígenas; el GIA (Grupo de Investigaciones Agrarias), centro dedicado al estudio y el fortalecimiento de las comunidades rurales, y SUR, dedicado a los estudios sociales vinculados, fundamentalmente, a los temas de pobreza, tanto urbano como rurales.

Estos centros aportaron con docentes (José Bengoa, Francisco Vergara y Miguel Bahamondes, entre los principales), trabajos de campo, prácticas profesionales y, por supuesto, tesis de grado, marcando con ello cierta impronta temática, por ejemplo, la ruralidad, lo indígena, la marginalidad y la pobreza. A esto, por supuesto, contribuyó la presencia temprana de otros docentes que no venían de estos centros, pero que poseían un perfil asociado a tales temas y que tuvieron incidencia en el desarrollo de las tesis realizadas: José Luis Martí- nez, Francisca Márquez, Pedro Mege, Andrea Seelenfreund, Viviana Manríquez, Elías Padilla y Luis Campos, entre otros ${ }^{8}$.

\section{La Escuela de Antropología y sus tesis}

La Escuela de Antropología de la Universidad Academia de Humanismo Cristiano, como ya se mencionó, fue creada en 1992 y tuvo como fundador y primer director a José Bengoa, quien por aquellos años, y entre otras tareas, dirigía la Comisión Especial de Pueblos Indígenas (CEPI), comisión gubernamental encargada de crear la Ley Indígena promulgada en 1993. Según palabras del propio Bengoa, la idea de crear un nuevo programa de formación antropológica obedecía a la necesidad de contar con un enfoque y un compromiso más fuerte de la antropología con las demandas y luchas de los pueblos indígenas (Espinoza, Campos \& Contreras, 2019). Es decir, y en relación con nuestra investigación, había en la escuela, desde un inicio, y en directa relación con el contexto local, una orientación bastante clara.

Al término de la dirección de José Bengoa, le sucedieron José Luis Martínez (1996-2001), Pedro Mege (2001-2007), Luis Campos (20072011), Claudio Espinoza (2011-2018) y Marinka Núñez (2018- presente), directores y directora que tienen en los temas indígenas y rurales sus principales líneas de trabajo. Por supuesto, esta fuerte orientación no excluyó la posibilidad de nuevas temáticas, pues tal cual ha sido señalado, la escuela siempre mantuvo el objetivo "de formar profesionales integrales, no sesgados ni en lo teórico ni en lo temático, por tanto, que ha evitado sucumbir a la especialización" (Espinoza, 2014, p. 11). 
Hasta enero de 2019, la Escuela de Antropología, cuenta con un total de 285 titulados y tituladas, quienes han conseguido el grado académico de licenciado(a) en antropología y el título profesional de antropólogo(a) a través de la confección de una tesis de grado.

\section{Metodología}

En consonancia con determinadas líneas de la sociología de la ciencia (Ramos, 2008), este artículo se inscribe en los estudios que se enfocan en la observación de las disciplinas científicas a través del análisis de los textos científicos, artículos o libros y, en nuestro caso, tesis. En este sentido, pretendemos aproximarnos a una de las experiencias de la formación antropológica en Chile a partir del estudio de las tesis producidas en una escuela en particular.

Esta producción científica coincide con el período de posdictadura, momento en que las disciplinas científicas experimentaron un crecimiento en sus recursos institucionales y en el que el campo recuperó una significativa legitimidad social (Palestini, Ramos \& Canales, 2010). En esta etapa, además, persisten una serie de tensiones sociales que permean, indudablemente, la producción científica. Por lo mismo, las tesis analizadas pueden dar cuenta de cómo una determinada experiencia de formación antropológica se vincula a la realidad bajo determinados contextos en el marco de los procesos históricos. Desde esta óptica, las tesis se conforman como parte de un capital científico que, a su vez, es parte de las condiciones sociales de producción de una sociedad y, por ende, se constituye en un espacio de lucha atravesado históricamente por diversos intereses (Bourdieu, 2003).
Bajo estas consideraciones, este artículo tiene como objetivo realizar una acercamiento descriptivo al quehacer de las ciencias antropológicas en Chile a partir de datos preliminares de un trabajo de sistematización y análisis de las tesis de grado de la Escuela de Antropología de la Universidad Academia de Humanismo Cristiano, realizadas en un período de veinte años, entre 1998 y 2018.

El levantamiento de los datos se realizó a partir de la revisión de un total de 241 tesis mediante una pauta que consideró 20 variables asociadas tanto a los atributos de los(as) autores(as) como a las características del propio texto científico. Para este artículo, las variables consideradas fueron: título de la tesis (temática), sexo, año, lugar de realización de la investigación (país, región, área geográfica) y zona (urbana/rural).

Como primer avance de este estudio se propone una clasificación de las tesis a partir de tres líneas generales de estudio dadas por la temática y la zona donde se desarrollan: i) estudios indígenas, ii) estudios de/en la ciudad (con exclusión de los temas indígenas) y, iii) estudios en comunidades rurales (campesinas, pesqueras y mineras). Agregamos una cuarta categoría dada por: iv) otros tipos de antropología, que incluye temáticas como género, creencias y otras, que tienen una presencia en la escuela cuantitativamente menor.

La información obtenida fue ordenada, sistematizada y recodificada con base en criterios que permitieran categorizar cada unidad de análisis. A partir de la aplicación de técnicas estadísticas se procedió, en una primera etapa, a la construcción de frecuencias para cada una de las variables, las cuales fueron analizadas de manera colectiva con el equipo de trabajo teniendo en consideración ciertas hipótesis iniciales para, posteriormente, proceder a un análisis multivariable. 


\section{Las tesis y algunas de sus características}

Entre 1998 y 2018 se han producido 241 tesis de antropología. En ellas han participado 285 estudiantes. Por tanto, y a pesar de que repre- sentan un gran número, no todas las tesis han sido confeccionadas individualmente. Hay 41 tesis (17\%) que han sido realizadas por más de una persona y 200 (83 \%) de elaboración individual.

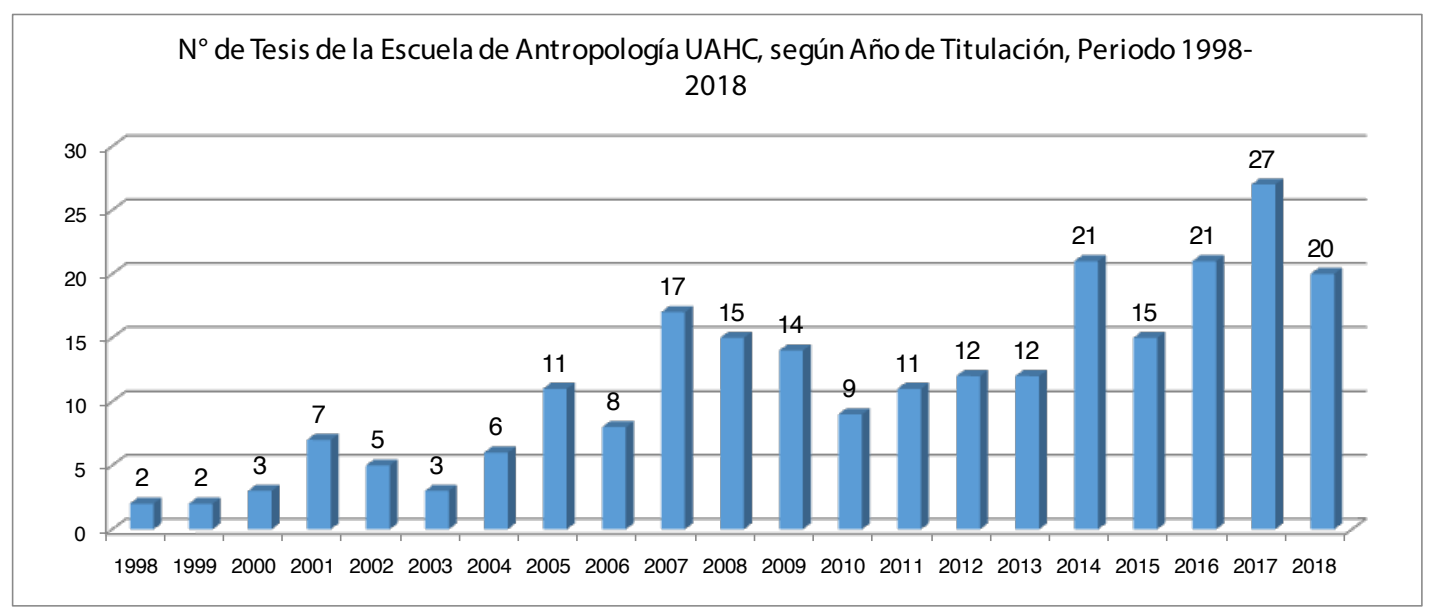

De los(as) 285 tesistas, 180 son mujeres (63\%) y 105, hombres (37\%).

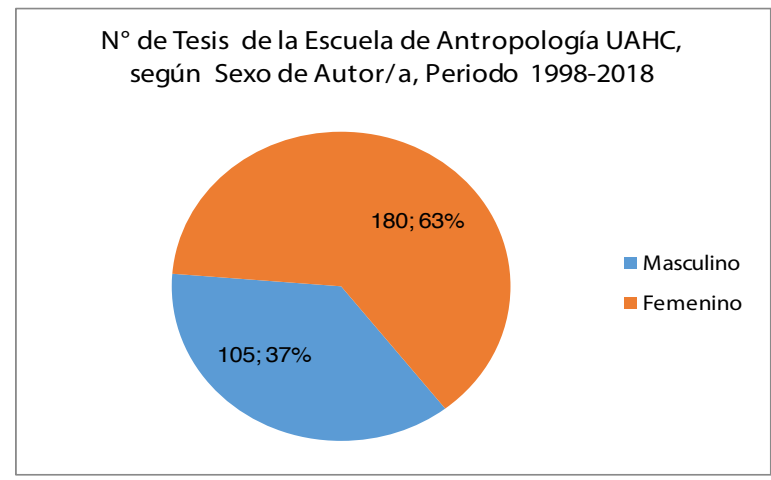

En términos de las regiones de estudio, las tesis revisadas muestran que la mayor parte $(55,6 \%)$ fueron realizadas sobre regiones tanto del norte como del sur del país, mientras que $37,8 \%$ se enfocaron en la Región Metropolitana, tanto en sus zonas rurales como urbanas. Por último, en $6,6 \%$ de las tesis no aplica la categoría de región, puesto que se trata de tesis teóricas, históricas, de evaluación de programas a nivel país o sobre análisis de obras artísticas, por ejemplo, cómics o la obra de Violeta Parra. 
№ de Tesis de la Escuela de Antropología UAHC según Región de Desarrollo de Estudio, Región Metropolitana y otras regiones

Periodo 1998 - 2018

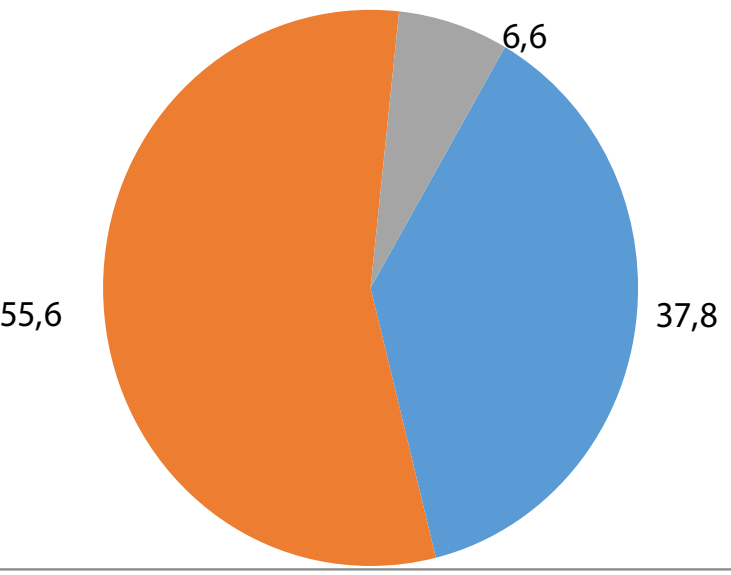

Región Metropolitana

55,6

37,8

- Otras Regiones del País

No Aplica

En cuanto a las regiones del país, las tesis se dividen de la siguiente manera:

№ de Tesis de la Escuela de Antropología UAHC según Región de Desarrollo de Estudio, Periodo 1998 - 2018

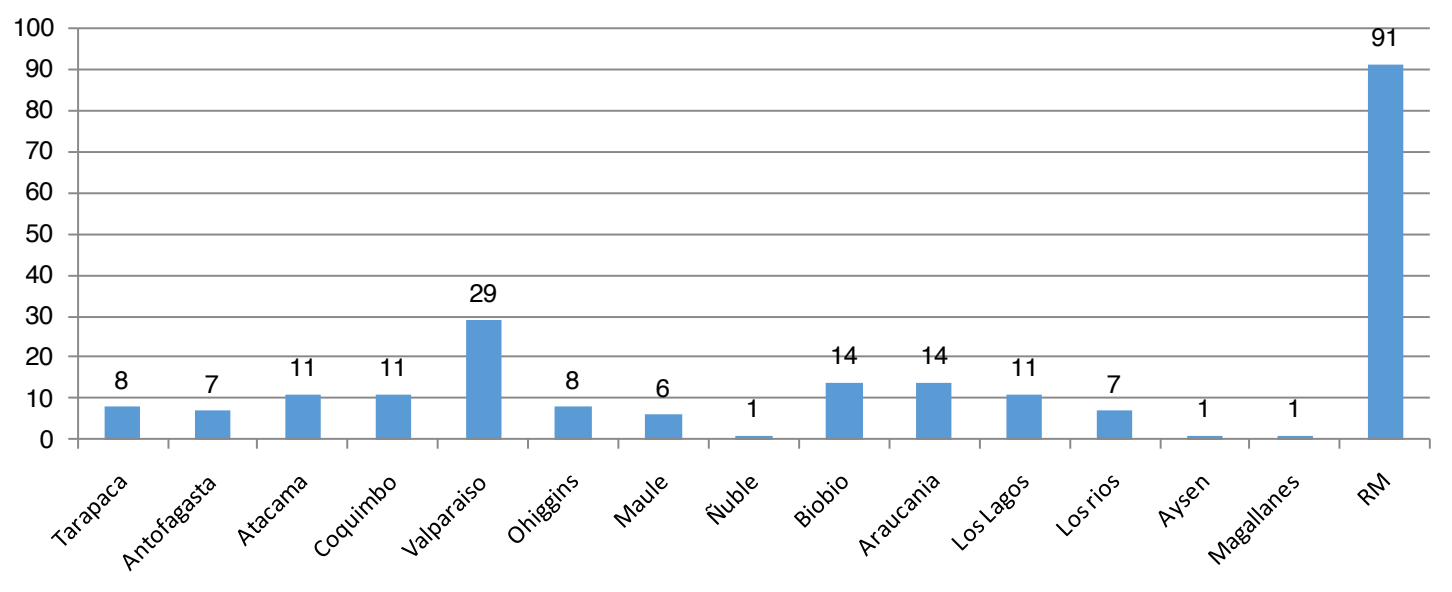

Agrupadas por zonas geográficas, las tesis presentan la siguiente distribución: el mayor porcentaje se lleva a cabo en la zona central del país, que incluye a la Región Metropolitana, la de Valparaíso por el norponiente, y las de O'Higgins, Maule y Ñuble por el sur.
Le sigue la zona sur, que incluye las regiones del Biobío, Araucanía, Los Lagos y Los Ríos, es decir, el corazón del territorio histórico mapuche. 
Luego están dos zonas geográficas contiguas, el Norte Chico y el Norte Grande, zonas fuertemente rurales, indígenas, pesqueras y mineras.
Por último, está el extremo sur, zona de muy difícil acceso. De hecho, solo dos tesis se han realizado en esas regiones. Y por último están las tesis realizadas en otros países, que son tres.

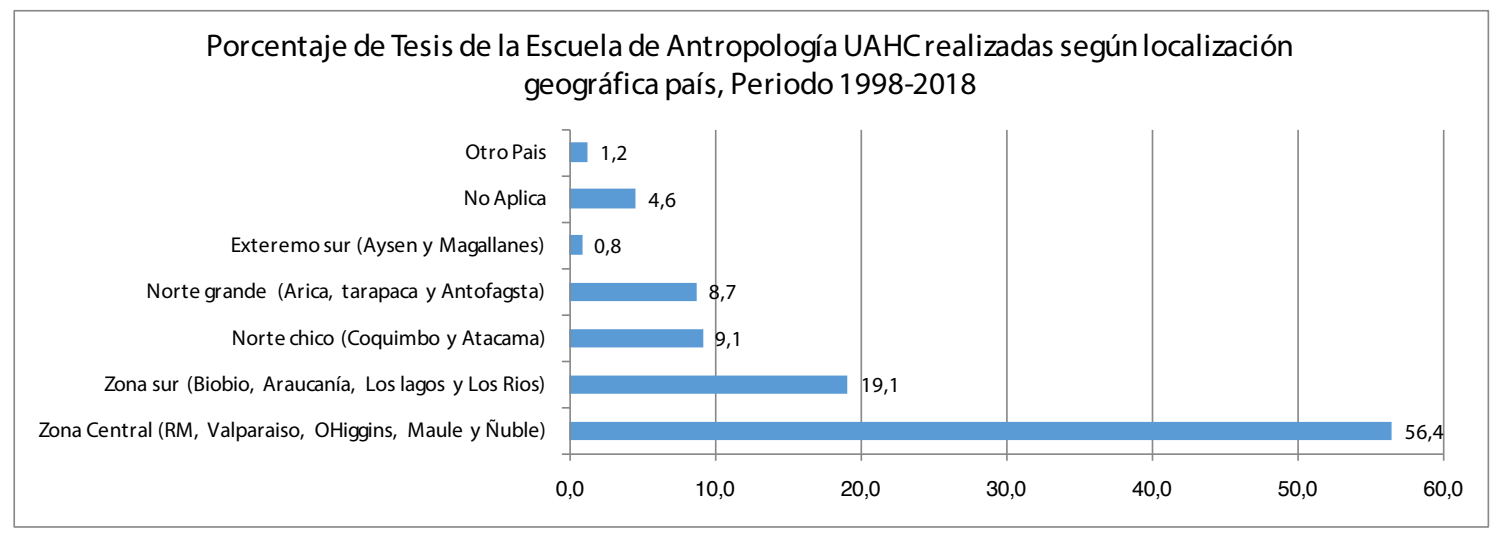

En términos de áreas rurales o urbanas, las tesis se dividen en dos: $50 \%$ en zonas rurales y $50 \%$ en zonas urbanas, con una leve ventaja (una tesis) para esta última categoría.

Por último, en cuanto a las temáticas abordadas, las tesis muestran ciertas tendencias fuertes. Una gran cantidad aborda temáticas relacionadas con los pueblos indígenas (30\%) y con comunidades rurales (31\%). Luego hay $23 \%$ que aborda temas insertos en la ciudad (excluyendo los temas étnicos). Falta analizar con mayor detalle estos últimos trabajos, pero desde ya se puede apreciar una inclinación hacia los temas de pobreza y marginalidad urbana.

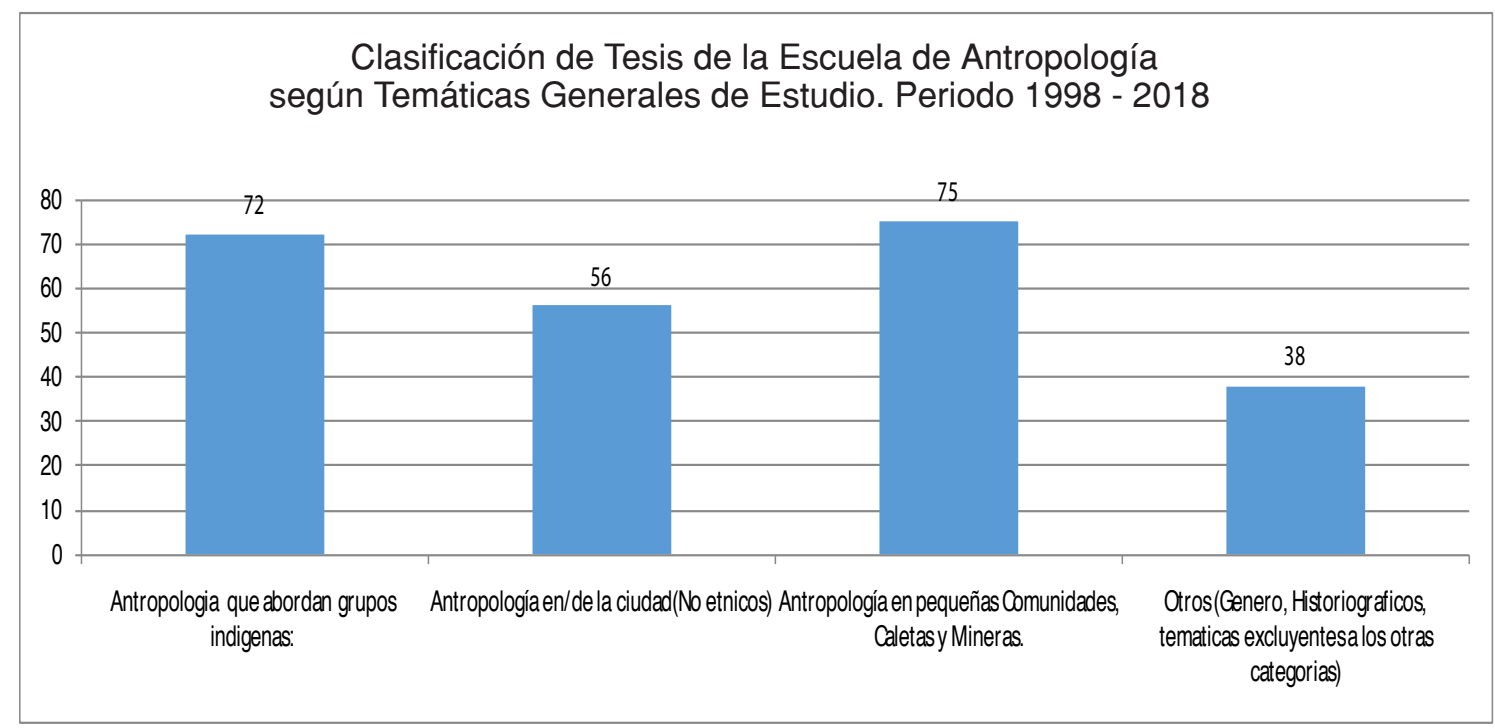


En el nivel que llevamos de investigación es prematuro sacar conclusiones, pero desde ya podemos advertir una relación en la temática de las tesis con los centros, los docentes, los diagnósticos y las proyecciones que estuvieron en la base de la creación de la Escuela de Antropología de la Universidad Academia de Humanismo Cristiano, esto es, temáticas relacionadas con los pueblos indígenas, con las comunidades rurales y con los temas de pobreza y marginalidad.

\section{Consideraciones finales}

Este avance de resultados permite sostener algunas consideraciones útiles para el debate en torno a la producción de conocimiento antropológico.

En primer lugar, la tesis, esta literatura gris, menos citadas y apreciadas que libros y artículos, constituye sin duda un aporte a la producción de conocimiento que, por un lado, aporta al caudal de conocimiento antropológico y, por el otro, puede dar cuenta de ciertas características de experiencias concretas en el amplio universo de las antropologías del sur.

En este sentido, la experiencia comparada y coordinada de este tipo de trabajos, por ejemplo, articulada a Grupos de Trabajo de la Asociación Latinoamericana de Antropología (ALA), bien pueden contribuir a la visibilización de estas antropologías no hegemónicas.

En segundo lugar, y siguiendo las iniciativas propuestas por Krotz (2006), recurrir a las trayectorias históricas para buscar en aquellos momentos iniciales la dialéctica entre las hipotecas exógenas y las innovaciones endógenas, puede resultar útil para establecer la relación entre, por una parte, las dinámicas políticas y el contexto local, los proyectos de formación antropológica, centros de estudios y equipos docente involucrados y, por la otra, la producción científica, en este caso, reflejada en las tesis de grado, particularmente a partir de sus ejes temáticos. En este sentido, el caso de la Escuela de Antropología de la Universidad Academia de Humanismo Cristiano es un buen reflejo de esta relación.

En tercer lugar, el análisis de las tesis de grado aquí esbozado, aporta datos interesantes y que llaman a la proliferación de estos ejercicios de modo de poder establecer análisis comparados y características generales de tipos de antropologías, locales, regionales, nacionales, o de mayor amplitud.

Algunos de los datos preliminares que arroja nuestro trabajo son los siguientes:

1) Se observa una mayor titulación de mujeres por sobre hombres: $63 \%$ por sobre $37 \%$,

2) De las 241 tesis analizadas, el 93,3 \% se llevó a cabo a través de un trabajo de campo en alguna localidad concreta, cuya cobertura alcanza todas las regiones del país, desde el extremo norte hasta el extremo sur,

3) En términos de áreas urbanas y rurales, las tesis se dividen en partes iguales entre ambas opciones.

4) En términos de las temáticas abordadas en las tesis, se observa una clara preponderancia de algunos temas por sobre otros: el $30 \%$ de las tesis abordan temas relacionados con pueblos indígenas; un 31\% aborda temas con comunidades rurales, campesinas, pesqueras o mineras, superando entre ambas el $60 \%$. 
5) Esta constatación encuentra sentido al relacionar la producción de tesis de grado, y el proyecto formativo de la Escuela analizada, sobre todo pensando en los centros que sirvieron de base para la constitución de la Escuela, así como en el temprano equipo de docentes y sus temas de trabajo.

6) Resta analizar el $39 \%$ restante, dividido en un $23 \%$ que aborda temas urbanos, con exclusión de los temas indígenas, y un 16\% categorizado por ahora como otros temas. Una mirada inicial a este $23 \%$ urbano, permite vislumbrar que una buena parte de dichos temas, abordan la desigualdad, pobreza y marginalidad urbana. Es decir, muestra coherencia con el momento inicial de la Escuela.

Por otro lado, la constatación de las inclinaciones temáticas aquí reseñadas, permite vislumbrar la posibilidad de algunos análisis comparativos a nivel nacional que deben ser, en todo caso, revisados y completados. Por ejemplo, un estudio de Skewes (2004), donde se hace una clasificación de 279 tesis producidas hasta el año 2004 en la Universidad de Chile, Universidad Austral de Chile, Universidad Bolivariana y Universidad Católica de Temuco, muestra que, con excepción de esta última, el tema indígena es minoritario. En un sentido similar, el trabajo de Arnold, Haefner, Quiroz, \& Radrigán (1990), muestra que, entre las 84 tesis producidas hasta la época del estudio en la Universidad de Chile, solo el $22 \%$ de los temas abordan cuestiones relacionadas con los pueblos indígenas.

El programa formativo en antropología de la Universidad Academia de Humanismo Cristiano representa entonces un caso diferente, con un porcentaje de temas que abordan los temas relacionados con los pueblos indígenas superior a lo mostrado en los estudios mencionados en el párrafo anterior.

Resta por cierto un largo y complejo análisis en nuestra investigación. Entre otros aspectos, profundizar en los temas abordados en el conjunto de tesis producidas. En una investigación realizada por Palestini, Ramos \& Canales (2010), se señala que la producción de la obra antropológica en el Chile de la post dictadura muestra una dispersión temática. Sería interesante ver si acaso tal observación se repite en las tesis de grado. Por ahora los datos aquí analizados muestran que las tesis de grado, al menos en grandes ejes temáticos, muestran ciertas tendencias relativamente claras.

\section{Notas}

\footnotetext{
${ }^{1}$ En https://antropotesis.alterum.info/index.php.

2 Ídem.

${ }^{3}$ Estas ideas han sido expuestas por Roberto Melville en diversas instancias de comunicación personal y colectivas

${ }^{4}$ Probablemente la fecha de 2004 corresponda más bien al término del estudio del autor, puesto que la primera versión de ese trabajo, publicada en inglés, data de 2005: "Social sciences and society in Chile: Institutionalization, breakdown and rebirth in Chile".

${ }^{5}$ Fases propuestas en Garretón (2007).

${ }^{6}$ Probablemente los programas abiertos durante el siglo XXI puedan corresponder a una nueva fase. No lo sabemos. Sí creemos que sería interesante conocer las características de estas nuevas escuelas
}

\footnotetext{
y departamentos de manera de situarlas en relación/comparación con los programas creados con anterioridad.

${ }^{7}$ Fases propuestas en Garretón (2007).

${ }^{8}$ Hubo, por cierto, otros docentes, como Rossana Cassigoli y Rainer Hausser, que estuvieron desde el comienzo en la Escuela, pero que no alcanzaron a dirigir tesis de grado. Y hay, por supuesto, un conjunto de docentes que tuvieron ingreso posterior a la Escuela y que han aportado (hasta 2018) significativamente en la producción de tesis, entre ellos, y por orden descendente, Claudio Espinoza, Mayarí Castillo, Daniel Quiroz, Carmen Gloria Godoy, Francisca Fernández, Claudia Arellano, Eduardo Sarué y Francisca Pérez.
} 


\section{Referencias bibliográficas}

Arnold, M., Haefner, C., Quiroz, D. \& Radrigán, M. (1990). Antropología social en Chile: Producciones y representaciones. Santiago de Chile: Universidad de Chile.

Bengoa, J. (2014). La trayectoria de la antropología en Chile. Antropologías del Sur, 1(1), 15-42.

Bourdieu, P. (2003). El oficio del científico: Ciencia de la ciencia y reflexividad. Barcelona: Anagrama

Cardoso de Oliveira, R. (1999). Antropologías periféricas "versus" antropologías centrales. En Conferencias y participaciones de investigadores invitados al $V$ Congreso argentino de Antropología Social. La Plata, Argentina, 19-37.

Castro, M. (2014). A sesenta años de la antropología en Chile. Antropologías del Sur, 1(1), 43-64.

Correa, M. (1993). Breve esbozo de la antropología brasileña reciente (1960-1980). Alteridades, 3(6), 13-16.

Espinoza, C. (2014). Presentación. Antropologías del Sur, 1(1), 11-14.

Espinoza, C., Campos, L., \& Contreras, P. (2019). Ciclo de conversaciones Antropologías del Sur: José Bengoa. Antropologías del Sur, 6(11), 225-248.

Díaz-Crovetto, G. (2011). Antropologías de las antropologías: buscando ciertas condiciones para su emergencia y consolidación. Antípoda, (12), 191-210.

Garretón, M. A. (2007). Las ciencias sociales en Chile: Institucionalización, rupturas y renacimiento. En Trindade, H. (Coord.), Las ciencias sociales en América Latina: Perspectiva comparada (pp. 193-248). México: Siglo XXI.

Gatti, P. \& De Souza, L. (2018). Presentación: Un prólogo para los diálogos de las antropologías latinoamericanas. En Gatti, P. \& De Souza, L. (Eds.), Diálogos con la antropología latinoamericana (pp. 7-12). Montevideo: Asociación Latinoamericana de Antropología (ALA).

Guber, R. (2006). Linajes ocultos en los orígenes de la antropología social de Buenos Aires. Avá Revista de Antropología, (8), 1-35

Jimeno, M. \& Arias, D. (2011). La enseñanza de la antropología en Colombia: Una antropología ciudadana. Alteridades, 21(41), 27-44.
Krotz, E. (1993). La producción de la antropología en el sur: Características, perspectivas, interrogantes. Alteridades, 3(6), 5-11. (2006). La diversificación de la antropología universal a partir de las antropologías del sur. Boletín Antropológico, 24(66), 7-20 (2015). Las antropologías segundas en América Latina: Interpelaciones y recuperaciones. Cuadernos de Antropología Social, (42), 5-17.

Lins Ribeiro, G. \& Escobar, A. (2008). Antropologías del mundo: Transformaciones disciplinarias dentro de sistemas de poder. En Lins Ribeiro, G. \& Escobar, A (Eds.), Antropologías del mundo: Transformaciones disciplinarias dentro de sistemas de poder (pp. 19-48), México: The Wenner-Gren International, Centro de Investigaciones y Estudios Superiores en Antropología Social, Envión.

Mora, H. (2014). Descentrar las miradas. Institucionalización de la antropología académica en la sede Temuco de la Pontificia Universidad Católica de Chile (1970-1978). Tabula Rasa, (21), 197-227 (2016). La institucionalización de las ciencias antropológicas en Chile: Una aproximación a las dinámicas socio-organizativas en la conformación del espacio científico (1860-1954). (Tesis inédita de doctorado en ciencias sociales). Universidad Nacional de La Plata, La Plata.

Palestini, S., Ramos, C. \& Canales, A. (2010). La producción de conocimiento antropológico social en Chile postransición: Discontinuidades del pasado y debilidades presentes. Estudios Atacameños, (39), 101-120.

Peirano, M. (1991). The anthropology of anthropology: The Brazilian case. Serie Antropologia, № 110. Brasilia: Universidade de Brasilia.

Ramos, C. (2008). ¿Sistema, campo de lucha o red de traducciones y asociaciones?: Tres modelos para investigar la ciencia social y un intento de integración. Persona y Sociedad, 22(2), 9-52.

Restrepo, E. (2014). Antropología hecha en Colombia. Antropologías del Sur, 1(1), 83-104.

Skewes, J. C. (2004). La enseñanza superior de la antropología en Chile. Anales del Instituto de Chile, XXIV(2), 357-399.

Vásquez, L. (2014). Ciento cuatro años de antropología mexicana. Antropologías del Sur, 1(1), 119-131. 